ACM International Conference Proceeding Series9 April 2018, Pages 59-642nd International Conference on Information System and Data Mining, ICISDM 2018; Florida Polytechnic UniversityLakeland; United States; 9 April 2018 through 11 April 2018; Code 137526

\title{
Quality data extraction methodology based on the labeling of coffee leaves with nutritional deficiencies(Conference Paper)
}

- Jungbluth, A.Email Author,

- Yeng, J.L.Email Author,

- Vives, L.Email Author

- Department of Computer Science, School of Engineering, Universidad

Peruana de Ciencias Aplicadas, Lima, Peru

\begin{abstract}
View references (13)
Nutritional deficiencies detection for coffee leaves is a task which is often undertaken manually by experts on the field known as agronomists. The process they follow to carry this task is based on observation of the different characteristics of the coffee leaves while relying on their own experience. Visual fatigue and human error in this empiric approach cause leaves to be incorrectly labeled and thus affecting the quality of the data obtained. In this context, different crowdsourcing approaches can be applied to enhance the quality of the data extracted. These approaches separately propose the use of voting systems, association rule filters and evolutive learning. In this paper, we extend the use of association rule filters and evolutive approach by combining them in a methodology to enhance the quality of the data while guiding the users during the main stages of data extraction tasks. Moreover, our methodology proposes a reward component to engage users and keep them motivated during the crowdsourcing tasks. The extracted dataset by applying our proposed methodology in a case study on Peruvian coffee leaves resulted in $93.33 \%$ accuracy with 30 instances collected by 8 experts and evaluated by 2 agronomic engineers with background on coffee leaves. The accuracy of the dataset was higher than independently implementing the evolutive feedback strategy and an empiric approach which resulted in $86.67 \%$ and $70 \%$ accuracy respectively under the same conditions. (C) 2018 Association for Computing Machinery.
\end{abstract}

SciVal Topic Prominence

Topic: Industry | Information systems | social media Prominence percentile: 90.273

Author keywords 
Data extractionData quality assessmentQuality data extraction methodology

Indexed keywords

Engineering controlled terms:

Engineering uncontrolled terms

Association rulesCrowdsourcingExtractionFiltrationInformation systemsInfor

Data extractionData quality assessmentFeedback strategiesHuman errorsQual systems

Engineering main heading: Data mining

- ISBN: 978-145036354-9

- Source Type: Conference Proceeding

- Original language: English

- DOI: $10.1145 / 3206098.3206102$

- Document Type: Conference Paper

- Sponsors:

- Publisher: Association for Computing Machinery 